\title{
O efeito da intervenção não farmacológica na densidade mineral óssea de pacientes com lesão medular: uma revisão sistemática
}

\section{The effect of non-pharmacological intervention on bone mineral density in patients with spinal cord injury: a systematic review}

Rickella Aparecida Alves Moreira ${ }^{1}$, (D) Natália Silveira de Paiva ${ }^{1}$, (D) Flavio Rodrigo Cichon ${ }^{1}$, (iD) Marta Imamura ${ }^{1}$, (iD) Daniel Rubio de Souza ${ }^{1}$

1 Instituto de Medicina Física e Reabilitação, Hospital das Clinicas da Faculdade de Medicina da Universidade de São Paulo

\section{Correspondência}

Rickella Aparecida Alves Moreira

E-mail: rickella.moreira@gmail.com

\section{Submetido: 8 Agosto 2020}

Aceito: 2 Março 2021

Como citar

Moreira RAA, Paiva NS, Cichon FR, Imamura M, Souza DR. O efeito da intervenção não farmacológica na densidade mineral óssea de pacientes com lesão medular: uma revisão sistemática. Acta Fisiatr. 2021;28(1):54-65.

10.11606/issn.2317-0190.v28i1a173528

2021 by Acta Fisiátric

\begin{abstract}
RESUMO
Objetivo: Avaliar os efeitos das medidas não farmacológicas na prevenção de perda ou no aumento da densidade mineral óssea de pacientes com lesão medular. Método: Revisão sistemática usando as bases de dados Medline, Embase, Cochrane Library e Lilacs com pesquisa entre 2009 e junho de 2019. Resultados: Foram encontrados 801 artigos dos quais foram selecionados, de acordo com os critérios de inclusão e exclusão, 15 artigos: 8 Estudos Clínicos Randomizados (ECR), 5 Estudos de Intervenção e 2 Revisões Sistemáticas. Conclusão: As evidências do uso de terapias não farmacológicas para prevenção e tratamento da osteoporose em LM são fracas e seus estudos contêm inúmeros vieses impossibilitando conclusões definitivas. O FES é a modalidade mais estudada e seu uso pode ser benéfico tanto para prevenção como para o tratamento da perda de massa óssea. Os resultados são observados com um uso frequente ( 5 sessões semanais) e associado a atividades físicas em especial as que promovam maior resistência muscular. Destacamos também que o retardo na perda de massa óssea está circunscrito ao período de aplicação, cessando após o término do mesmo. A associação de eletroestimulação e exercícios parece potencializar a ação medicamentosa, mas mais estudos são necessários para ratificar esta impressão. Intervenções não farmacológicas como o ortostatismo, atividades físicas, treino de marcha e a eletroestimulação são estratégias de baixo custo, baixo risco, poucos efeitos colaterais e com inúmeros outros benefícios na reabilitação de lesados medulares. Por isso, ainda que não tenhamos evidências consistentes de ação na massa óssea, estão fortemente recomendados.
\end{abstract}

Palavras-chaves: Traumatismos da Medula Espinal/reabilitação, Osteoporose, Densidade Óssea, Exercício Físico, Estimulação Elétrica

\section{ABSTRACT}

Objective: To evaluate the effects of non-pharmacological measures (exercise, orthostatism, electrical stimulation, gait training, vibrating platform and physical activity) in preventing loss or increasing bone mineral density in patients with spinal cord injury. Method: Systematic review using the Medline, Embase, Cochrane Library and Lilacs databases with research between 2009 and June 2019. Results: 801 articles were found from which 15 articles were selected, according to the inclusion and exclusion criteria: 8 Randomized Clinical Studies (RCT), 5 Intervention Studies and 2 Systematic Reviews. Conclusions: Evidence of the use of non-pharmacological therapies for the prevention and treatment of osteoporosis in $\mathrm{SCl}$ is weak and its studies contain numerous biases making definitive conclusions impossible. FES is the most studied modality. Its use can be beneficial for both prevention and treatment of bone loss. The results are observed with frequent use ( 5 weekly sessions) and associated with physical activities, especially those that promote greater muscular resistance. The delay in the loss of bone mass is limited to the application period, stopping after the end of the application. The association of electrostimulation and exercise seems to enhance the medication action, but more studies are needed to confirm this impression. Nonpharmacological interventions such as orthostatism, physical activities, gait training and electrostimulation are low-cost, low-risk, have few side effects and a numerous other benefits in the rehabilitation of $\mathrm{SCl}$. Therefore, although we do not have consistent evidence of action on bone mass, they are strongly recommended.

Keywords: Spinal Cord Injuries/rehabilitation, Osteoporosis, Bone Density, Exercise, Electric Stimulation 


\section{INTRODUÇÃO}

A lesão medular (LM) é uma condição clínica comum, com distribuição universal e com importante impacto funcional e expressivos custos para os sistemas de saúde.

A incidência anual de LM nos Estados Unidos é de 17.700 novos casos por ano, com média de idade de 43 anos no momento da lesão, sendo $78 \%$ das vítimas do gênero masculino. A prevalência é estimada de 288.000 pessoas. ${ }^{1}$ No Brasil, a incidência de trauma raquimedular é de 40 casos novos/ano/milhão de habitantes, ou seja, cerca de 6 a 8 mil casos novos por ano, sendo que destes $80 \%$ das vítimas são homens e $60 \%$ encontram-se entre 10 e 30 anos de idade. ${ }^{2}$

$\mathrm{O}$ indivíduo com lesão medular pode evoluir com inúmeras complicações clínicas e seu tratamento e manutenção implicam em grandes esforços em cuidados de saúde, medidas psicossociais e financeiras. A osteoporose, e o consequente risco de fratura óssea por fragilidade, é uma destas complicações.

Após a lesão observa-se uma rápida e severa diminuição na densidade mineral óssea (DMO $)^{3,4}$ podendo levar ao quadro de osteoporose em cerca de $81 \%$ dos casos. ${ }^{5}$ A osteoporose é uma doença óssea sistêmica caracterizada pela redução e deterioração da microarquitetura do tecido ósseo levando ao aumento da fragilidade óssea e a maior suscetibilidade à fraturas. ${ }^{6}$

A manutenção da homeostase óssea (preservação da força e elasticidade do tecido ósseo) depende da remodelação óssea, que substitui continuamente o osso antigo e danificado por um novo. Dois tipos de células estão envolvidos no processo de remodelação óssea: os osteoclastos (responsáveis pela reabsorção óssea) e os osteoblastos (responsáveis pela formação óssea). ${ }^{7} 0$ imobilismo secundário a LM combinado com fatores neurogênicos, circulatórios e hormonais resultam em um desbalanço na homeostase óssea, havendo maiores taxas de reabsorção do que formação óssea nas áreas abaixo da lesão. ${ }^{8,9}$ Além disso, outras condições como idade, sexo, nível e tempo de lesão, capacidade de deambular, espasticidade, nutrição, privação do sol, abuso de medicamentos como corticoides, heparina e anticonvulsivantes podem contribuir para um risco aumentado de perda óssea e osteoporose. ${ }^{10}$

A desmineralização ocorre principalmente nas áreas sublesionais e predominantemente nos locais de sustentação de peso, como o fêmur distal e a tíbia proximal, que são ricos em osso trabecular. Em áreas diafisárias do fêmur e da tíbia, com predomínio de osso cortical, o tecido é relativamente poupado. ${ }^{8,9}$ Isso pode explicar por que o fêmur distal e a tíbia proximal são locais frequentes de fratura nesta população. ${ }^{11}$

Em contrapartida, a coluna vertebral não parece ser afetada pela desmineralização. O aumento do estresse na coluna causado pela permanência prolongada em cadeira de rodas pode ter um efeito osteogênico nas vértebras e, assim, contribuir para a manutenção ou aumento da DMO vertebral. Para os membros superiores, o resultado depende do nível da lesão: apenas pacientes tetraplégicos apresentam uma diminuição da DMO nos braços e antebraços. ${ }^{12}$

A perda óssea começa logo após a lesão e continua a uma taxa de aproximadamente $4 \%$ ao mês em regiões com osso trabecular predominante e $2 \%$ ao mês em locais que contêm principalmente osso cortical. Esse processo é intenso nos primeiros 6 meses $^{13}$ e atinge um platô aos 16 meses pós-lesão, o que equivale a uma diminuição da DMO de $50 \%$ em comparação aos valores pré lesão nesses pacientes. ${ }^{12,14}$

A taxa de perda óssea após a LM é substancialmente maior do que as observadas em outras condições tais como imobilização no leito por período prolongado $(0,1 \%$ por semana), osteoporose pós menopausa na ausência de tratamento medicamentoso (3-5\% por ano) e condições de microgravidade como voos no espaço ( $0,25 \%$ por semana). ${ }^{15,16}$

As fraturas geralmente envolvem a tíbia ou a fíbula, mas também podem acometer a extremidade superior, mais comumente naqueles com lesões medulares mais altas.

Geralmente ocorrem por atividades de baixo impacto como transferência de cama para cadeira e vice-versa, manobras incorretas de transferência, mas também podem resultar de acidentes de trânsito e queda da cadeira de rodas. ${ }^{13,15}$ Sabe-se que a incidência de fraturas aumenta em função do tempo de lesão, com cerca de $1 \%$ ao ano de incidência em indivíduos com menos de 10 anos de lesão, e 3,4\% a 4,6\% de fraturas por ano em pessoas com mais de 10 anos de lesão. ${ }^{17}$

Os fatores de risco para fraturas por fragilidade após LM incluem: sexo feminino (as mulheres correm maior risco em comparação aos homens), tempo após a lesão (o risco de fratura aumenta significativamente aos 10 anos após a lesão), idade que o indivíduo sofreu a lesão (maior risco para aqueles com lesões antes dos 16 anos de vida), gravidade da deficiência (lesões completas têm maior perda), índice de massa corporal baixo, DMO da região distal do fêmur e uso de anticonvulsivantes, heparina ou analgésicos opióides. ${ }^{18}$

As fraturas pós LM, podem resultar em atraso ou não consolidação óssea, formação de pseudoartrose e gerar complicações secundárias a imobilização como trombose venosa profunda, deformidades, piora da espasticidade, dor, crises de hipertensivas por disreflexia autonômica e lesão por pressão (que pode resultar em osteomielite e até amputações). Esses fatores aumentam o custo das despesas médicas e da morbi-mortalidade ${ }^{18,19}$ justificando assim a importância do estudo de formas de prevenção e tratamento da osteoporose nesta população.

Os exames de imagem comumente usados para quantificar a DMO são a absorciometria de raios $X$ de dupla energia (DXA), a tomografia computadorizada quantitativa (QCT), a tomografia computadorizada quantitativa periférica ( $p Q C T)$ e a ressonância magnética (RM), sendo o primeiro mais frequentemente utilizado na prática clínica e o últimos mais utilizados em pesquisas clínicas pois não tem padronização populacional como a DXA. ${ }^{16,18}$ Importante ressaltar que na interpretação desses exames temos que avaliar não apenas se houve ganho de massa óssea, mas também se houve estabilização ou diminuição na velocidade de perda óssea considerando este um efeito positivo da intervenção.

Em relação ao tratamento farmacológico, existem várias opções no mercado como bifosfonatos, raloxifeno, ranelato de estrôncio, teriparatida e denosumab. Porém, em LM, há poucos estudos sendo a classe de droga mais estudada e utilizada os bifosfonados. $^{8}$

As intervenções não farmacológicas, como ortostatismo, exercícios físicos, treino de marcha (com ou sem equipamentos), estimulação elétrica (EE), ultrassom 
terapêutico (US) e suplementação dietética (em especial cálcio e vitamina D) podem ser importantes estratégias de prevenção e tratamento da osteopenia/osteoporose do ponto de vista de custo, poucos efeitos adversos e contraindicações e outro benefícios associados as outros acometimentos clínicos relacionados a LM. O caráter multifatorial da osteoporose nestes indivíduos também reforça o potencial valor adjuvante destas terapias.

Em 2009, Biering-Sørensen et al. ${ }^{20}$ publicaram uma revisão sistemática que avaliou o efeito das intervenções não farmacológicas na DMO em pacientes com LM, avaliando a influência de descarga de peso em ortostatismo e caminhada, exercícios, eletroestimulação funcional (FES), campos eletromagnéticos, vibração, US e ocorrência de espasticidade.

A baixa densidade mineral óssea em LM ainda é um problema pouco estudado e com poucas soluções terapêuticas eficazes. Portanto, frente aos novos avanços da medicina, fazse necessária nova revisão sistemática. Os dados obtidos neste estudo, serão comparados aos obtidos pela revisão realizada em 2009.

\section{OBJETIVO}

Considerando-se o impacto da morbimortalidade causada pela osteoporose em pacientes com LM, torna-se de extrema relevância a prevenção e tratamento desta condição. Desta forma, o presente estudo tem como objetivo realizar uma revisão sistemática da literatura analisando o efeito das intervenções não farmacológicas na DMO de pacientes com LM traumática.

\section{MÉTODOS}

Esta revisão sistemática foi realizada de acordo com os Itens de Relatórios Preferenciais para Revisões Sistemáticas e MetaAnálises de Protocolos (PRISMA-P). Foram revisados artigos publicados desde o ano de 2009, quando foi publicada a seguinte revisão sistemática " Non-pharmacological treatment and prevention of bone loss after spinal cord injury: a systematic review "que serviu de base para a realização deste estudo. A revisão sistemática em questão, foi publicada na revista Spinal Cord e está disponível para consulta na base de dados do Pubmed. ${ }^{20}$

A elaboração da pergunta científica realizada, foi baseada na estratégia PICO. Dessa forma, os artigos selecionados para a realização dessa revisão englobam pacientes com LM de origem traumática submetidos a intervenções não farmacológicas, sendo o desfecho primário analisado, a melhora da DMO. Para comparação, todas as demais intervenções existentes na literatura foram consideradas farmacológicas e não farmacológicas.

Foram selecionados estudos que incluíam humanos adultos, maiores que 18 anos de idade, com LM de causa traumática, sem limitação do tempo de lesão.

As intervenções consideradas neste estudo foram: marcha, ortostatismo, exercício físico, fisioterapia, estimulação elétrica funcional (FES), treino de marcha robotizada, plataforma vibratória, US, dieta, estilo de vida e uso de órteses. Foram incluídos revisões sistemáticas; ensaios clínicos controlados randomizados; ensaios clínicos controlados não randomizados.
O período selecionado foi de janeiro de 2009 a Junho de 2019, sendo considerados estudos publicados em língua inglesa, espanhola e portuguesa.

Coletados e dispostos em tabelas os dados relativos aos autores, nível de evidência do artigo, desenho do estudo, data de publicação e localidade (país) da publicação, número de pacientes utilizados no estudo, sexo dos pacientes, nível da LM, comorbidades, medicações em uso, intervenção avaliada, resultado obtido.

Foram excluídos estudos que incluíram intervenções feitas em animais, crianças e adolescentes (até os 18 anos); pessoas saudáveis sem LM; pacientes com LM de outras etiologias que não traumática. Estudos observacionais, cartas aos editores, relatos de caso, séries de caso, artigos incompletos não publicados e protocolos de pesquisa não foram considerados para esta revisão.

Inicialmente foram definidos os termos para a realização da pesquisa nas seguintes bases de dados: Medline (PubMed) www.pubmed.com; Biblioteca Cochrane www.thecochranelibrary.com; Lilacs (Literatura LatinoAmericana e do Caribe em Ciências da Saúde) www.lilacs.bvsalud.org e Embase - www.embase.com. Utilizouse os termos Medical Subject Headings $\left(\mathrm{MeSH}^{\circledR}\right)$, através da ferramenta MesH on Demand. A pesquisa foi realizada em Junho de 2019, em inglês e português.

Os MeSH Terms utilizados na pesquisa na base de dados PubMED foram os seguintes: Spinal Cord Injuries; Spinal Cord Diseases; Paraplegia; Quadriplegia; Paraparesis; Paralysis; Physical Therapy Modalities; Exercise Therapy; Exercise; Resistance Training; Electric Stimulation; Electric Stimulation Therapy; Ultrasonic Waves; Ultrasonic Therapy; Ultrasonography, Interventional; Electromagnetic; Fields; Walking; Gait; Motor Activity; Posture; Weight-Bearing; Vibration; Compressive Strength; Diet; Life Style; Orthotic Devices; Robotics; Osteoporosis; Bone Diseases Metabolic ; Absorptiometry Photon; Bone Density.

Para pesquisa na base de dados Cochrane e Lilacs foram utilizados os seguintes termos: Spinal Cord Injuries; Spinal Cord Diseases; Paraplegia; Paraparesis; Paralysis; Physical Therapy Modalities; Exercise Therapy; Exercise; Resistance Training; Electric Stimulation; Electric Stimulation Therapy; Ultrasonic Waves; Ultrasonic Therapy; Ultrasonography, Interventional Electromagnetic Fields; Walking; Gait; Motor Activity; Posture; Weight-Bearing; Vibration; Compressive Strength; Diet; Life Style; Orthotic Devices; Robotics; Osteoporosis; Bone Diseases, Metabolic; Absorptiometry, Photon; Bone Density.

Já na plataforma de dados Embase, foram utilizados os Entree Terms descritos abaixo: Spinal Cord Injury; Spinal Cord Disease; Paraplegia; Quadriplegia; Paralysis; Physiotherapy; Kinesiotherapy; Exercise; Resistance Training; Electrostimulation; Electrotherapy; Ultrasound; Ultrasound Therapy; Interventional; Ultrasonography Electromagnetism; Walking; Gait; Motor Activity; Body position; Weight Bearing; Vibration; Compressive Strength; Diet; Lifestyle; Orthosis; Robotics; Osteoporosis; Metabolic bone disease; Photon absorptiometry; Bone Density.

Realizada a busca de artigos, seguido pela exclusão de estudos duplicados e seleção de artigos duplicados por dois autores independentes (NSP e RAAM). 
As discordâncias foram resolvidas por consenso e, em caso de discordância, um terceiro autor foi consultado (DRS). Todos os estudos foram analisados segundo seus títulos e resumos, de acordo com critérios de inclusão e exclusão. Uma vez preenchidos os critérios de elegibilidade, realizou-se a leitura na íntegra dos artigos selecionados. Todos os estudos com texto completo avaliados foram descritos na seção "Resultados". Dados faltantes foram esclarecidos entrando em contato direto com os autores.

Esta revisão sistemática é relatada baseando-se nas diretrizes do PRISMA. O detalhamento do processo de busca e extração de dados estão disponibilizados no fluxograma (Figura 1).

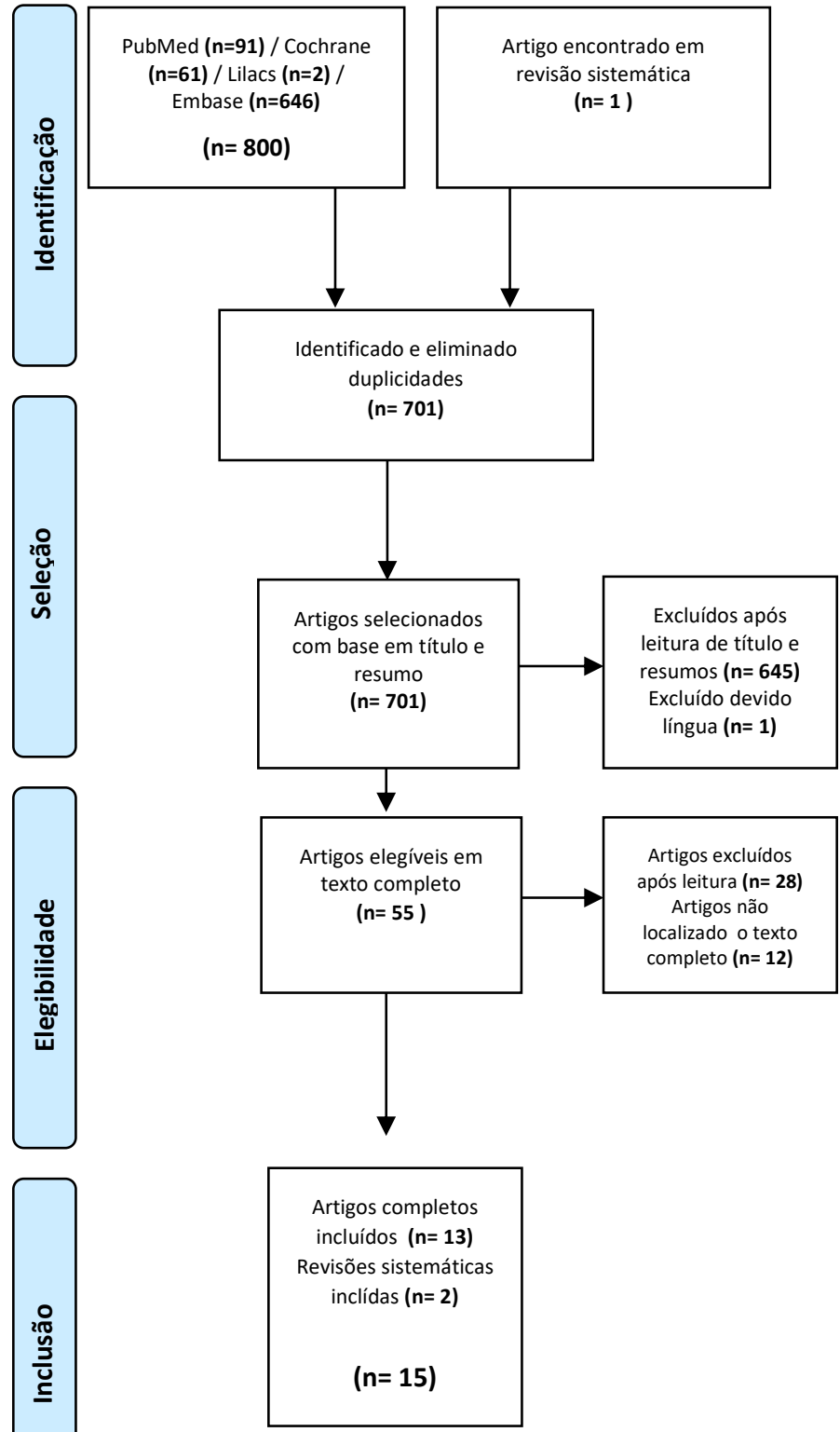

Figura 1. Fluxograma das diferentes fases da revisão

Foram utilizadas as diretrizes do Grupo Cochrane Back Review para análise dos artigos de intervenção selecionados. Tais diretrizes avaliam viés de seleção, performance, detecção, atrito, relato e outras fontes de viés menos frequentes.

\section{RESULTADOS}

De todos os 801 artigos encontrados através da nossa estratégia de pesquisa, foram selecionados, de acordo com os critérios de inclusão, 15 artigos para a revisão sistemática. Sendo 8 Estudos Clínicos Randomizados (ECR), 5 Estudos de Intervenção e 2 Revisões Sistemáticas. Vale ressaltar que foram checados individualmente os artigos destas duas revisões sistemáticas.

Caso não estivessem inclusos em nosso levantamento, o seriam. Porém, esses artigos não foram encontrados na integra, impossibilitando a sua inclusão no presente trabalho. Estas revisões tiveram seus resultados comparados com os encontrados em nosso trabalho e estes serão discutidos nas conclusões do mesmo. Os artigos selecionados foram avaliados quanto ao risco de viés e estes dados estão dispostos no Quadro 1.

Quadro 1. Riscos de viés

\begin{tabular}{|c|c|c|c|c|c|c|c|}
\hline Autor/Ano & 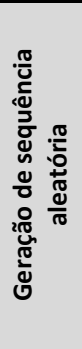 & 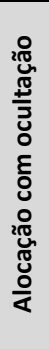 & 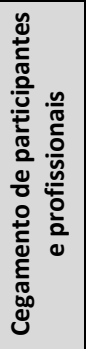 & 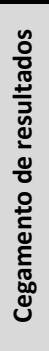 & 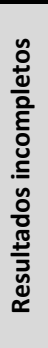 & 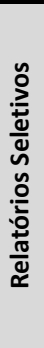 & 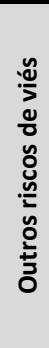 \\
\hline $\begin{array}{l}\text { Craven et al. }{ }^{21} \\
2017\end{array}$ & V & $\mathrm{V}$ & $x$ & V & $x$ & $x$ & $x$ \\
\hline $\begin{array}{l}\text { Arija-Blázquez et al. }{ }^{22} \\
2014\end{array}$ & V & $\mathrm{V}$ & $x$ & $\mathrm{~V}$ & $\mathrm{~V}$ & $\mathrm{~V}$ & V \\
\hline $\begin{array}{l}\text { Menéndez et al. }{ }^{23} \\
2016\end{array}$ & $?$ & $?$ & $x$ & V & V & $\mathrm{V}$ & $?$ \\
\hline $\begin{array}{l}\text { Dudley-Javoroski et al. }{ }^{24} \\
2012\end{array}$ & $x$ & $x$ & $x$ & V & $?$ & $?$ & $x$ \\
\hline $\begin{array}{l}\text { Groah et al. } .^{25} \\
2010\end{array}$ & V & $?$ & $?$ & $\mathrm{~V}$ & $\mathrm{~V}$ & $\mathrm{~V}$ & $?$ \\
\hline $\begin{array}{l}\text { Lai et al. }{ }^{26} \\
2010\end{array}$ & $?$ & $?$ & $?$ & $?$ & $?$ & $\mathrm{~V}$ & $?$ \\
\hline $\begin{array}{l}\text { Morse et al. }{ }^{27} \\
2019\end{array}$ & $?$ & $?$ & $?$ & $?$ & $x$ & $\mathrm{~V}$ & $?$ \\
\hline $\begin{array}{l}\text { Dudley-Javoroski et al. }{ }^{28} \\
2016\end{array}$ & $x$ & $x$ & $x$ & V & $x$ & $\mathrm{~V}$ & $?$ \\
\hline $\begin{array}{l}\text { Edwards et al. }{ }^{29} \\
2018\end{array}$ & $?$ & $?$ & V & $?$ & V & $\mathrm{V}$ & $x$ \\
\hline $\begin{array}{l}\text { Wuermser et al. }{ }^{30} \\
2015\end{array}$ & $x$ & $x$ & $x$ & V & V & $\mathrm{V}$ & $?$ \\
\hline $\begin{array}{l}\text { Astorino et al. }{ }^{31} \\
2013\end{array}$ & $x$ & $x$ & $x$ & V & $x$ & $\mathrm{~V}$ & $?$ \\
\hline $\begin{array}{l}\text { Chain et al. }{ }^{32} \\
2012\end{array}$ & $x$ & $x$ & $x$ & V & V & $\mathrm{V}$ & $x$ \\
\hline $\begin{array}{l}\text { Karelis et al. }{ }^{33} \\
2017\end{array}$ & $x$ & $x$ & $x$ & $x$ & V & V & $?$ \\
\hline
\end{tabular}

Baixo risco de viés $=V \quad$ Viés Incerto $=$ ?

Foram extraídos os principais dados, conforme demonstrados no Quadro 2. Os estudos analisados separados pelo tipo de intervenção, serão apresentados nos Quadros 2 e 3 e posteriormente, no Quadro 4 serão apresentadas as limitações dos estudos analisados. 
Quadro 2. Dados gerais

\begin{tabular}{|c|c|c|c|c|c|c|c|c|}
\hline $\begin{array}{l}\text { Autor / } \\
\text { Estudo }\end{array}$ & Local & $\begin{array}{l}\text { Design do } \\
\text { Estudo }\end{array}$ & $\begin{array}{l}\text { Idade / } \\
\text { Sexo }\end{array}$ & $\begin{array}{l}\text { Tipo e Tempo da } \\
\text { Lesão Medular }\end{array}$ & $\begin{array}{l}\text { Intervenção } \\
\text { estudada }\end{array}$ & $\begin{array}{c}\text { Duração / } \\
\text { intervenção }\end{array}$ & $\begin{array}{l}\text { Seguimento } \\
\text { longitudinal / } \\
\text { intervenção }\end{array}$ & $\begin{array}{c}\text { Forma de } \\
\text { avaliação } \\
\text { da DMO }\end{array}$ \\
\hline $\begin{array}{l}\text { Craven et al. }{ }^{21} \\
2017\end{array}$ & Canadá & ECR & $\begin{array}{l}26 \mathrm{H} \\
\text { Média: 56,59 anos } \\
\text { (intervenção); } \\
\text { 54,06 controle }\end{array}$ & $\begin{array}{l}\text { AIS C ou D, C2 a t12 } \\
\text { Maior ou igual a } 18 \mathrm{~m}\end{array}$ & FES & 4 meses & 12 meses & $\begin{array}{l}\text { DXA e } \\
\text { pQCT }\end{array}$ \\
\hline $\begin{array}{l}\text { Arija-Blázquez } \\
\text { et al. }{ }^{22} 2014\end{array}$ & Espanha & ECR & $\begin{array}{l}12 \mathrm{H} \\
18 \text { a } 55 \text { anos }\end{array}$ & $\begin{array}{l}\text { AIS A, T4 a T12 } \\
<\text { de } 8 \text { semanas }\end{array}$ & FES & 14 semanas & 14 semanas & DXA \\
\hline $\begin{array}{l}\text { Menéndez et } \\
\text { al. }^{23} 2016\end{array}$ & Espanha & ECR & $\begin{array}{l}12 \mathrm{H}, 5 \mathrm{M} \\
\text { Média: } 49,9 \text { anos }\end{array}$ & $\begin{array}{l}\text { AIS A ou B, C4 a L1 } \\
\text { Média de 13,3 anos } \\
\text { após lesão }\end{array}$ & Vibração + FES & 12 semanas & 20 semanas & DXA \\
\hline $\begin{array}{l}\text { Dudley-Javoroski } \\
\text { et al. }{ }^{24} 2011\end{array}$ & EUA & $\begin{array}{l}\text { Estudo de } \\
\text { Intervenção }\end{array}$ & $\begin{array}{l}36 \mathrm{H}, 7 \mathrm{M} \\
16 \text { a } 64 \text { anos } \\
(28 \mathrm{LM}, 14 \mathrm{~s} / \mathrm{LM})\end{array}$ & AIS A e B, C5 a T12 & $\begin{array}{l}\text { Estimulação } \\
\text { Elétrica unilateral } \\
+ \text { Treinamento } \\
\text { quadríceps + } \\
\text { Ortostatismo }\end{array}$ & $\begin{array}{l}\text { Não } \\
\text { mencionado }\end{array}$ & $\begin{array}{l}\text { Não } \\
\text { mencionado }\end{array}$ & pQCT \\
\hline $\begin{array}{l}\text { Groah et al. }{ }^{25} \\
2010\end{array}$ & EUA & ECR & $\begin{array}{l}22 \text { H, } 4 \text { M } \\
\text { Média: } 26,2 \text { anos } \\
\text { (intervenção) } \\
\text { 31,1 anos } \\
\text { (controle) }\end{array}$ & $\begin{array}{l}\text { AIS A e B, acima de T12 } \\
\text { Menos de } 12 \text { semanas } \\
\text { de lesão }\end{array}$ & $\begin{array}{l}\text { Atividade física + } \\
\text { EE }\end{array}$ & 6 semanas & $\begin{array}{l}3 \text { meses após } \\
\text { intervenção }\end{array}$ & DXA \\
\hline $\begin{array}{l}\text { Lai et al. }{ }^{26} \\
2010\end{array}$ & Taiwan & ECR & $\begin{array}{l}20 \text { H, } 4 \text { M } \\
\text { Média: } 28,9 \text { anos } \\
\text { (intervenção) } \\
28,3 \text { (controle) }\end{array}$ & $\begin{array}{l}\text { AIS A, C5 a T10 } \\
26 \text { a } 52 \text { dias após lesão }\end{array}$ & $\begin{array}{l}\text { FES + Bicicleta } \\
\text { ergométrica }\end{array}$ & 6 meses & 6 meses & DXA \\
\hline $\begin{array}{l}\text { Morse et al. }{ }^{27} \\
2019\end{array}$ & EUA & ECR & $\begin{array}{l}18 \mathrm{H}, 2 \mathrm{M} \\
\text { Média de idade: } \\
38,2\end{array}$ & $\begin{array}{l}\text { AIS A, B ou C } \\
\text { Nível a partir de C4 } \\
\text { Lesão de } 18 \text { meses } \\
\text { ou mais }\end{array}$ & $\begin{array}{l}\text { FES + remo X FES } \\
\text { + remo + ácido } \\
\text { zoledronico }\end{array}$ & 12 meses & 12 meses & vQCT \\
\hline $\begin{array}{l}\text { Dudley-Javoroski } \\
\text { et al. }{ }^{28} 2016\end{array}$ & EUA & $\begin{array}{l}\text { Estudo de } \\
\text { Intervenção }\end{array}$ & $\begin{array}{l}5 \mathrm{H}, 2 \mathrm{M} \\
17 \text { anos a } 66 \text { anos }\end{array}$ & $\begin{array}{l}\text { AIS A ou B } \\
0,1 \text { a } 29,2 \text { anos } \\
\text { após lesão }\end{array}$ & Vibração & 12 meses & 12 meses & $\begin{array}{l}\text { pQCT e } \\
\text { TCAR }\end{array}$ \\
\hline $\begin{array}{l}\text { Edwards et al. }{ }^{29} \\
2018\end{array}$ & EUA & ECR & $\begin{array}{l}45,4 \text { média total de } \\
\text { idade }\end{array}$ & $\begin{array}{l}\text { AIS A, B, C, D } \\
\text { Todos os níveis cervical, } \\
\text { torácico, lombar } \\
\text { LM }<1 \text { ano da } \\
\text { randomização }\end{array}$ & $\begin{array}{l}\text { GRUPO 1: } \\
\text { Teriparatida + } \\
\text { vibração simulada } \\
\text { GRUPO 2: } \\
\text { Placebo + vibração } \\
\text { GRUPO 3: } \\
\text { Teriparatida + } \\
\text { vibração }\end{array}$ & 12 meses & 24 meses & DXA e TC \\
\hline $\begin{array}{l}\text { Wuermser et } \\
\text { al. }^{30} 2015\end{array}$ & EUA & $\begin{array}{l}\text { Estudo de } \\
\text { Intervenção }\end{array}$ & $\begin{array}{l}6 \mathrm{H}, 6 \mathrm{M} \\
25 \text { a } 50 \text { anos }\end{array}$ & $\begin{array}{l}\text { AIS A ou B } \\
2 \text { a } 27 \text { anos após LM }\end{array}$ & $\begin{array}{l}\text { Ortostatismo + } \\
\text { plataforma } \\
\text { vibratória }\end{array}$ & 6 meses & 12 meses & $\begin{array}{l}\text { DXA e } \\
\text { pQCT }\end{array}$ \\
\hline $\begin{array}{l}\text { Astorino et al. }{ }^{31} \\
2013\end{array}$ & EUA & $\begin{array}{l}\text { Estudo de } \\
\text { Intervenção }\end{array}$ & $\begin{array}{l}11 \mathrm{H}, 2 \mathrm{M} \\
19 \text { a } 42 \text { anos }\end{array}$ & $\begin{array}{l}\text { Lesão completa ou } \\
\text { incompleta, Níveis } \\
\text { abaixo de C2 } \\
\text { Lesão aguda } \\
\text { (<3anos) e crônica } \\
\text { (>3anos) }\end{array}$ & $\begin{array}{l}\text { Atividade física, } \\
\text { vibração, bicicleta } \\
\text { ergométrica, } \\
\text { marcha, FES }\end{array}$ & 6 meses & 6 meses & $\mathrm{DX}$ \\
\hline $\begin{array}{l}\text { Chain et al. }{ }^{32} \\
2012\end{array}$ & Brasil & $\begin{array}{l}\text { Estudo de } \\
\text { intervenção }\end{array}$ & $\begin{array}{l}25 \mathrm{H} \text { - } 15 \text { grupo } \\
\text { ativos } \\
10 \text { sedentários } \\
19 \text { a } 56 \text { anos }\end{array}$ & $\begin{array}{l}\text { Entre C5 e C7 } \\
\text { Lesão completa e } \\
\text { incompleta }\end{array}$ & $\begin{array}{l}\text { Atividade fisica } \\
\text { habitual }\end{array}$ & $\begin{array}{l}\text { Não } \\
\text { mencionado }\end{array}$ & $\begin{array}{l}\text { Não } \\
\text { mencionado }\end{array}$ & DXA \\
\hline $\begin{array}{l}\text { Karelis et al. }{ }^{33} \\
2017\end{array}$ & Canadá & $\begin{array}{l}\text { Estudo de } \\
\text { Intervenção }\end{array}$ & $\begin{array}{l}4 \mathrm{H}, 1 \mathrm{M} \\
\text { Média: } 60,4 \text { anos }\end{array}$ & $\begin{array}{l}\text { AIS A, C7 a T10 } \\
\text { Média de } 7,6 \text { anos } \\
\text { após lesão }\end{array}$ & $\begin{array}{l}\text { Exoesqueleto } \\
\text { robótico (EKSO - } \\
\text { Ekso Bionics } \\
\text { Richmond) }\end{array}$ & 6 semanas & $\begin{array}{l}\text { Não } \\
\text { mencionado }\end{array}$ & DXA \\
\hline
\end{tabular}

Absorciormetria de dupla energia por dupla energia por raio x (DXA); Densidade mineral óssea (DMO); Eletroestimulação (EE); Eletroestimulação funcional (FES); Ensaio clínico randomizado (ECR); Homens (H); Lesão Medular (LM); Mulheres (M); Tomografia computadorizada quantitativa periférica (pQCT) 
Quadro 2. FES e intervenções combinadas

\begin{tabular}{|c|c|c|c|c|c|}
\hline Intervenção & Autor & Grupo controle & Grupo intervenção & Medição da DMO & Resultados \\
\hline \multirow[t]{2}{*}{ 岃 } & $\begin{array}{l}\text { Craven et al. } \\
2017\end{array}$ & $\begin{array}{l}\text { N inicial= } 17 \text { participantes } \\
\mathrm{N} \text { finall } 12 \text { participantes } \\
\text { Exercício aeróbicos: } 20 \text { a } 25 \text { min. } \\
\text { Escala Borg } 3 \text { a } 5 \\
\text { Exercícios resistidos supervisionados: } 2 \text { a } 3 \text { séries de } 12 \\
\text { a } 15 \text { repetições resistência máxima para músculos com } \\
\text { contração voluntária } \\
\text { No de sessões: não foi possível calcular } \\
\mathrm{N} \text { inicial= } 17 \text { participantes } \\
\mathrm{N} \text { final= } 12 \text { participantes } \\
\text { Exercício aeróbicos: } 20 \text { a } 25 \text { min. } \\
\text { Escala Borg } 3 \text { a } 5 \\
\text { Exercícios resistidos supervisionados: } 2 \text { a } 3 \text { séries de } 12 \\
\text { a } 15 \text { repetições resistência máxima para músculos com } \\
\text { contração voluntária } \\
\text { № de sessões: não foi possível calcular }\end{array}$ & $\begin{array}{l}\text { N inicial= } 17 \text { participantes } \\
\mathrm{N} \text { final= } 16 \text { participantes } \\
\text { Caminhada assistida + FES de } 45 \mathrm{~min}, 3 \mathrm{x} \\
\text { por semana, por } 4 \text { meses } \\
\text { Amplitude de pulso } 8 \text { a } 125 \mathrm{~mA} \\
\text { Duração de pulso } 0 \text { a } 300 \text { us } \\
\text { Frequências de } 20 \text { a } 50 \mathrm{~Hz} \\
\text { № de sessões: } 48 \\
\text { Local estimulado: Quadríceps, } \\
\text { isquiotibiais, tibial anterior e } \\
\text { gastrocnêmios }\end{array}$ & $\begin{array}{l}\text { DXA de quadril esquerdo (quadril total), } \\
\text { fêmur distal direito e tíbia proximal direita } \\
\text { pQCT de extremidade distal e o eixo da tíbia } \\
\text { para obter informações sobre osso } \\
\text { trabecular e osso cortical, respectivamente } \\
\text { DXA e pQCT em } 0,4 \text { e } 12 \text { semanas }\end{array}$ & $\begin{array}{l}\text { Não houve diferença } \\
\text { significativa na DMO entre os } \\
\text { grupos, visto que a alteração } \\
\text { mínima considerada } \\
\text { significativa para o DXA foi de } \\
2 \% \text { no fêmur distal e de } 3 \% \text { no } \\
\text { tíbia proximal }\end{array}$ \\
\hline & $\begin{array}{l}\text { Arija-Blázquez } \\
\text { et al. }{ }^{22} 2014\end{array}$ & $\begin{array}{l}\mathrm{N} \text { inicial= } 5 \text { participantes } \\
\mathrm{N} \text { final= } 3 \text { participantes } \\
\text { Protocolo realizado similar ao grupo intervenção, exceto } \\
\text { pela amplitude máxima de } 0 \mathrm{~mA} \\
\text { № de sessões: } 70\end{array}$ & $\begin{array}{l}N \text { inicial= } 7 \text { participantes } \\
N \text { final= } 5 \text { participantes } \\
1 \text { sessão= } 80 \text { contrações musculares } \\
\text { durante } 47 \text { min, divididos em } 10 \text { séries } \\
\text { com } 60 \text { segundos de descanso entre elas } \\
5 \text { dias/semana, durante } 14 \text { semanas } \\
\text { Amplitude máxima de } 140 \mathrm{~mA} \\
\text { Duração de pulso de } 200 \text { us } \\
\text { Frequência de } 30 \mathrm{~Hz} \\
\text { № de sessões: } 70 \\
\text { Local estimulado: Reto femoral, Vasto } \\
\text { medial lateral }\end{array}$ & $\begin{array}{l}\text { DXA de pernas, na região lombar e nos } \\
\text { quadris, considerando } \\
\text { todo o quadril, o colo do fêmur, as áreas } \\
\text { trocantérica e intertrocantérica e o } \\
\text { triângulo de Ward } \\
\text { DXA em } 0 \text { e } 14 \text { semanas }\end{array}$ & $\begin{array}{l}\text { As porcentagens de alteração } \\
\text { na DMO de pré e pós- } \\
\text { intervenção não foram } \\
\text { significativamente diferentes } \\
\text { entre os dois grupos } \\
\text { A alteração mínima } \\
\text { considerada significativa para o } \\
\text { quadril inteiro foi de } 0,00963 \\
\mathrm{~g} / \mathrm{cm} 2 \text {, enquanto para a coluna } \\
\text { lombar foi de } 0,0806 \mathrm{~g} / \mathrm{cm} 2\end{array}$ \\
\hline 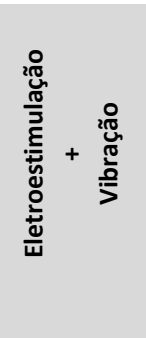 & $\begin{array}{l}\text { Menéndez et al. }{ }^{23} \\
2016\end{array}$ & $\begin{array}{l}N \text { inicial e final= } 8 \text { participantes } \\
\text { Treino de rotina= Todos foram submetidos a } 10 \\
\text { sessões/mês, com duração de } 2 \text { horas, de ortostatismo } \\
\text { ou inclinação, movimentos passivos, treinamento } \\
\text { resistido de baixa intensidade ou eletroterapia e } \\
\text { fisioterapia }\end{array}$ & $\begin{array}{l}N \text { inicial e final= } 9 \text { participantes } \\
\text { Sessões de } 10 \text { min de } P V+\text { EE durante } \\
12 \text { semanas aplicados antes dos treinos } \\
\text { de rotina } \\
\text { № de sessões: } 30 \\
\text { PV } \\
\text { Frequência de vibração } 10 \mathrm{~Hz} \\
\text { Amplitude } 5 \mathrm{~mm} \\
\text { Eletroestimulação } \\
\text { Duração de pulso } 400 \mu \text { s } \\
\text { Frequência } 8 \mathrm{~Hz} \\
\text { Amplitude inicial } 49,5+/-11,2 \mathrm{~mA} \\
\text { Localização: } 1 \text { eletrodo em fossa } \\
\text { poplítea e } 2 \text { em gastrocnêmios }\end{array}$ & $\begin{array}{l}\text { DXA do colo do fêmur } \\
\text { DXA em } 0 \text { e } 12 \text { semanas }\end{array}$ & $\begin{array}{l}\text { A DMO do colo do fêmur, } \\
\text { desfecho secundário, } \\
\text { permaneceu invariável durante } \\
\text { o estudo, tanto para grupo } \\
\text { controle quanto intervenção }\end{array}$ \\
\hline 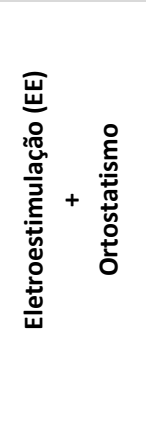 & $\begin{array}{l}\text { Dudley-Javoroski } \\
\text { et al. } .^{24} \\
2012\end{array}$ & $\begin{array}{l}\text { Grupo } 3 \\
\mathrm{~N}=16 \text { participantes } \\
0 \% \text { do peso corporal } \\
\text { Não realizaram ortostatismo, CR inclinada ou EE }\end{array}$ & $\begin{array}{l}\text { Grupo } 1 \text { (High Dose - ortostatismo + EE) } \\
-150 \% \text { do peso corporal } \\
\mathrm{N}=7 \text { participantes } \\
\text { Ortostatismo + estímulo em quadríceps } \\
\text { unilateral com joelho flexionado em } 20^{\circ} \\
20 \mathrm{~Hz} / / 200 \mathrm{us} / / \text { até } 200 \mathrm{~mA} \\
2 \text { sessões de EE com } 5 \mathrm{~min} \text { de descanso, } \\
5 \mathrm{x} / \text { semana } \\
\text { Alguns pacientes receberam CR e } \\
\text { fizeram treino domiciliar } \\
\text { № sessões estimado para os indivíduos } \\
\text { que realizaram por } 3 \text { anos: } 720 \\
\text { Grupo } 2 \text { (Low Dose - ortostatismo) - } 40 \% \\
\text { do peso corporal } \\
\mathrm{N}=5 \text { participantes } \\
\text { Ortostatismo ou em Cadeira de rodas } \\
\text { inclinada a } 900 \mathrm{SEM} \mathrm{EE} \\
30 \text { min, } 5 \times / \text { semana }\end{array}$ & pQCT em 0,1 e 3 anos & $\begin{array}{l}\text { Altas doses de carga em } \\
\text { ativação de quadríceps atenuou } \\
\text { significativamente o declínio da } \\
\text { DMO no fêmur distal quando } \\
\text { comparado aos outros grupos }\end{array}$ \\
\hline 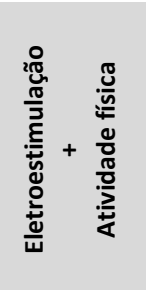 & $\begin{array}{l}\text { Groah et al. }{ }^{25} \\
2010\end{array}$ & $\begin{array}{l}\mathrm{N} \text { inicial }=13 \text { participantes } \\
\mathrm{N} \text { final }=10 \text { participantes } \\
\text { Terapia individualizada }\end{array}$ & 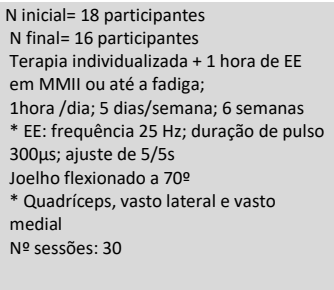 & $\begin{array}{l}\text { Osteocalcina } \\
\text { N-telopeptídeo na urina } \\
\text { DXA de coluna lombar e no colo do fêmur } \\
\text { bilateral, fêmur distal e tíbia proximal } \\
\text { As análises foram realizadas nos seguintes } \\
\text { momentos: } \\
\text { 10 Pré intervenção } \\
\text { 20 Pós-intervenção imediata } \\
\text { 30 3m pós-intervenção }\end{array}$ & $\begin{array}{l}\text { A DMO do grupo controle } \\
\text { diminuiu da seguinte forma: } \\
\text { lombar em } 1,88 \% \text {, quadril em } \\
12,25 \% \text {, fêmur distal } 15,15 \% \text { e } \\
\text { tíbia proximal em } 17,40 \% \text {. } \\
\text { Semelhante ao grupo controle, } \\
\text { o grupo EE demonstrou perdas } \\
\text { na DMO, como descrito a } \\
\text { seguir: lombar diminuiu } 1,29 \% \text {, } \\
\text { quadril } 14,45 \% \text {, fêmur distal } \\
7,40 \% \text {, e tíbia proximal em } \\
12,31 \%\end{array}$ \\
\hline 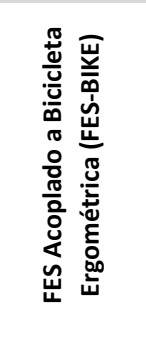 & $\begin{array}{l}\text { Lai et al. }{ }^{26} \\
2010\end{array}$ & $\begin{array}{l}\text { N inicial e final= } 12 \text { participantes } \\
\text { * Não relatado qual atividade desse grupo }\end{array}$ & 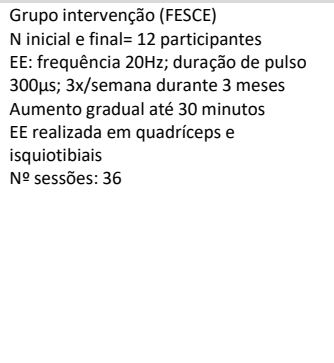 & $\begin{array}{l}\text { DXA de fêmur distal e colo de fêmur a } \\
\text { direita } \\
\text { Início (1a medição) } \\
\text { Após } 3 \text { meses ( } 2^{a} \text { medição) } \\
\text { Após } 3 \text { meses de interrupção do programa } \\
\text { FESCE ( } 3 \text { a medição) }\end{array}$ & $\begin{array}{l}\text { 1ạ medição: não houve } \\
\text { diferença entre os grupos } \\
\text { 2a medição: houve diminuição } \\
\text { da DMO em ambos os grupos, } \\
\text { porem menor no grupo } \\
\text { intervençãa: as taxas de } \\
\text { diminuição da DMO são } \\
\text { equivalentes a 2,23\% (DP 0,97) } \\
\text { para o grupo FESCE e 6,65\% (DP } \\
\text { 0,90) para o grupo controle } \\
\text { 3ạ medição: as DMO } \\
\text { apresentaram reduções } \\
\text { significativas em relação às } \\
\text { segundas medidas nos dois } \\
\text { grupos }\end{array}$ \\
\hline 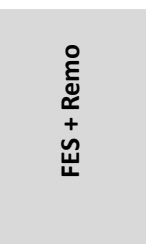 & $\begin{array}{l}\text { Morse et al. }{ }^{27} \\
2019\end{array}$ & $\begin{array}{l}\text { Grupo controle (FES + remo) } \\
\mathrm{N} \text { inicial= } 18 \text { participantes } \\
\mathrm{N} \text { final= } 10 \text { participantes } \\
\mathrm{EE} \text { : largura pulso } 450 \mathrm{~ms} \text {; Frequência } 40 \mathrm{~Hz} ; 6 \text { segundos } \\
\text { por contração } \\
\text { Quadríceps e isquiotibiais } \\
3 \text { a } 5 \text { dias/semana } \\
\text { Meta: } 30 \text { minutos, intensidade de } 75 \text { a } 85 \% \\
\text { da } \mathrm{FC} \\
\text { № total de sessões: variou de } 144 \text { a } 240\end{array}$ & $\begin{array}{l}\text { Grupo intervenção (FES + remo + AZ) } \\
N \text { inicial= } 20 \text { participantes } \\
N \text { final= } 10 \text { participantes } \\
\text { FES }+ \text { remo como descrito no grupo } \\
\text { controle }+1 \text { dose única }(5 \mathrm{~m} / 100 \mathrm{mg}) \text { de } \\
\text { AZ após treinamento de força, } 1 \text { ano } \\
\text { após início }\end{array}$ & $\begin{array}{l}\text { vQCT de fêmur distal e tíbia proximal } \\
\text { 1a medição (início) } \\
\text { 2 medição (12 meses após início) }\end{array}$ & $\begin{array}{l}\text { Tratamento com AZ atenuou } \\
2,5 \text { a } 8 \% \text { de perda de massa } \\
\text { óssea comparado com somente } \\
\text { FES-remo em LM crônica } \\
\text { O efeito do remo foi } \\
\text { detectável apenas na tíbia } \\
\text { proximal, era dependente da } \\
\text { dose e foi muito menor em } \\
\text { magnitude do que o efeito AZ }\end{array}$ \\
\hline
\end{tabular}




\section{Quadro 3. Outras intervenções}

\begin{tabular}{|c|c|c|c|c|}
\hline Intervenção & Autor & Grupo controle & Medição da DMO & Resultados \\
\hline & $\begin{array}{l}\text { Dudley-Javoroski } \\
\text { et al. }{ }^{28} \\
2016\end{array}$ & $\begin{array}{l}\mathrm{N} \text { inicial= } 7 \text { participantes } \\
\mathrm{N} \text { final= } 6 \text { participantes } \\
3 \text { participantes - vibração aplicada no MI dominante } \\
3 \text { participantes -vibração aplicada no } \mathrm{MI} \text { não } \\
\text { dominante } \\
112 \text { sessões de vibração ao longo de } 12 \text { meses, com } \\
\text { média > 2,14 sessões/semana }\end{array}$ & $\begin{array}{l}\text { PQCT de tíbia e fêmur distal } \\
\text { pQCT: Um a seis exames bilaterais de } \\
\text { pQCT, abrangendo até } 8,9 \text { anos após LM } \\
\text { TCAR: Um indivíduo foi submetido à } \\
\text { TCAR aos } 4 \text { meses e } 2,7 \text { anos após o LM } \\
\text { (participante } 1 \text { ) } \\
\text { Seis outros participantes foram } \\
\text { submetidos à TCAR antes e depois de } \\
\text { receber } 12 \text { meses de treinamento em } \\
\text { vibração }\end{array}$ & $\begin{array}{l}\text { Não foi observado melhora da DMO ou arquitetura } \\
\text { trabecular após } 12 \text { meses de treinamento vibratório } \\
\text { Em todas as regiões da TCAR, o declínio percentual } \\
\text { médio foi de } 29,5 \% \text { para a DMO }\end{array}$ \\
\hline 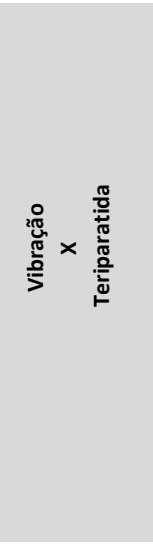 & $\begin{array}{l}\text { Edwards et al. }{ }^{29} \\
2018\end{array}$ & $\begin{array}{l}\text { Grupo } 1 \text { (Teriparatida } 20 \mu \mathrm{g} / \text { dia + Vibração simulada } \\
10 \mathrm{~min} / \text { dia) } \\
\mathrm{N} \text { inicial e final= } 20 \text { participantes } \\
\text { Grupo } 2 \text { (Placebo + Vibração } 10 \mathrm{~min} / \text { dia) } \\
\mathrm{N} \text { inicial = } 20 \text { participantes } / / \mathrm{N} \text { final= } 18 \\
\text { participantes } \\
\text { Vibração: Frequência } 30 \mathrm{~Hz} \\
\text { Ambos os pés apoiados na plataforma (individuo } \\
\text { sentado) } \\
\text { Grupo } 3 \text { (Teriparatida } 20 \mu \mathrm{g} / \text { dia + Vibração } \\
10 \mathrm{~min} / \text { dia) } \\
\mathrm{N} \text { inicial= } 21 \text { participantes } / / \mathrm{N} \text { final= } 18 \text { participantes } \\
\text { Vibração: Frequência } 30 \mathrm{~Hz} \\
\text { Ambos os pés apoiados na plataforma (individuo } \\
\text { sentado) } \\
* * \text { Em todos os grupos, duração de } 12 \text { meses, com } \\
\text { sessões diárias }\end{array}$ & DXA e TC em 0, 12 e 24 meses & $\begin{array}{l}\text { Na DXA, observou-se que a região epifisária } \\
\text { trabecular do fêmur apresentou uma perda de DMO } \\
\text { de } 24,2 \%(-57,5 \text { a 9,09) no grupo } 1 \text {, bem como uma } \\
\text { perda de } 14 \%(-47,8 \text { a } 18,9) \text { no grupo } 3 \text {, enquanto no } \\
\text { grupo } 2 \text { observou-se uma melhoria de } 10,3 \%(-21,1 \text { a } \\
41,7) \text {. No entanto, vale ressaltar que os resultados } \\
\text { não apresentaram significância estatística }\end{array}$ \\
\hline 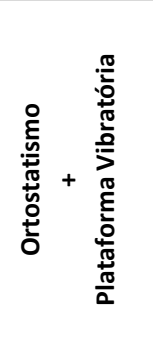 & $\begin{array}{l}\text { Wuermser } \\
\text { et al. }^{30} \\
2015\end{array}$ & $\begin{array}{l}\mathrm{N} \text { inicial= } 12 \text { participantes } / / \mathrm{N} \text { final= } 8 \text { participantes } \\
\text { Uso doméstico da plataforma vibratória }(34 \mathrm{~Hz} \text {, } \\
\text { movimento de } \sim 50 \mu \mathrm{m}) \text { : } \\
\text { Ortostatismo, durante } 20 \mathrm{~min} \\
5 \text { dias/semana, durante } 6 \text { meses } \\
\text { № sessões: } 120\end{array}$ & $\begin{array}{l}\text { DXA do fêmur proximal (quadril total e } \\
\text { colo do fêmur) } \\
\text { HRpQCT da tíbia distal não dominante } \\
4 \text { medidas de DXA e HRpQCT: } \\
\text { Inicial, em } 3 \text { e } 6 \text { meses durante a } \\
\text { intervenção, e novamente, } 6 \text { meses após } \\
\text { a interrupção da intervenção }\end{array}$ & $\begin{array}{l}\text { Não observada nenhuma melhora relevante na } \\
\text { densidade óssea ou na microestrutura após } 6 \text { meses e } \\
\text { nem no seguimento } \\
\text { A taxa de perda óssea no quadril foi inferior a } \\
\text { observada na tíbia } \\
\text { Quadril total: diminuição da DMO em } 1,4 \% \\
\text { Colo do fêmur: DMO mantida } \\
\text { Tíbia total: diminuição da DMO de } 4,59 \% \text {. } \\
\text { As taxas de perda óssea foram calculadas levando em } \\
\text { consideração o valor inicial e final (após } 6 \text { meses da } \\
\text { interrupção da intervenção) }\end{array}$ \\
\hline 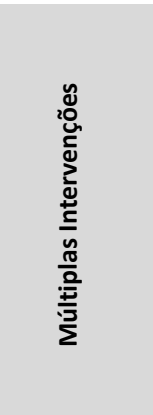 & $\begin{array}{l}\text { Astorino et al. }{ }^{31} \\
2013\end{array}$ & $\begin{array}{l}\text { Atividade Física + Vibração + Bicicleta + Marcha + FES } \\
N \text { inicial= } 13 \text { participantes } / / \mathrm{N} \text { final= } 10 \text { participantes } \\
\text { ATIVIDADE FíSICA: } 2 \text { a } 3 \text { horas/dia ABT, sendo } 2 \\
\text { dias/semana } \\
\text { VIBRAÇÃO: } 10 \text { a } 30 \text { minutos/semana } \\
\text { BICICLETA: } 15 \text { a } 30 \text { minutos de exercícios cíclicos ou } \\
\text { bicicleta ergométrica } \\
\text { MARCHA } \\
\text { Marcha passiva ( } N=5 \text { ): } 15 \text { a } 30 \text { minutos } \\
\text { Marcha ativa ( } N=8): 15 \text { a } 60 \text { minutos } \\
\text { FES: } 30 \text { minutos/semana, em quadríceps, glúteos e } \\
\text { isquiotibiais } \\
\text { Duração da intervenção: } 6 \text { meses }\end{array}$ & DXA realizada em 0, 3 e 6 meses & $\begin{array}{l}\text { A DMO total do corpo diminuiu } 2,5 \%(p=0,003) \text { de } 0 \text { a } \\
6 \text { meses, acompanhada de reduções significativas (- } \\
6,1 \%) \text { na DMO total do quadril }(p=0,001) \text { aos } 3 \text { e } 6 \\
\text { meses } \\
\text { A DMO no colo do fêmur direito }(-5,2 \%, p=0,001 \text { para } \\
\text { a direita e } p=0,04 \text { para a esquerda) declinou } \\
\text { consistentemente de } 0 \text { a } 6 \text { meses; enquanto a DMO } \\
\text { do colo do fêmur esquerdo diminuiu de } 0 \text { a } 3 \text { meses } \\
\text { após o qual foi mantida }\end{array}$ \\
\hline 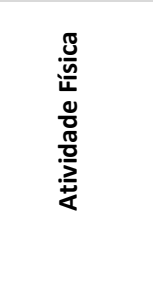 & $\begin{array}{l}\text { Chain et al. }{ }^{32} \\
2012\end{array}$ & $\begin{array}{l}\mathrm{N} \text { inicial= } 10 \text { participantes } \\
\text { Sem prática de atividade física após LM } \\
\text { Ativos } \\
\mathrm{N} \text { inicial= } 15 \text { participantes } \\
\text { Foram considerados ativos os que realizavam } \\
\text { exercícios com seguinte frequência: } \\
\text { Pelo menos } 150 \text { minutos/semana, em } 3 \text { dias/semana } \\
\text { por } 3 \text { meses consecutivos } \\
\text { № sessões: } 36\end{array}$ & DXA - realizada apenas 1 medida & $\begin{array}{l}\text { Resultados sugerem que o início precoce do exercício } \\
\text { físico após a ocorrência de lesão pode ajudar a } \\
\text { atenuar a desmineralização do osso fêmur em } \\
\text { homens com LM cervical }\end{array}$ \\
\hline 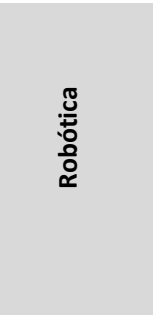 & $\begin{array}{l}\text { Karelis et al. }{ }^{33} \\
2017\end{array}$ & $\begin{array}{l}\text { Exoesqueleto Robótico } \\
\mathrm{N} \text { inicial e final= } 5 \text { participantes } \\
60 \text { minutos em caminhadas; } 3 \text { dias/semana; durante } \\
6 \text { semanas } \\
\text { Manutenção da intensidade de } 60 \text { a } 70 \% \text { FC } \\
\text { EKSO: composto por um par de pernas robótico } \\
\text { movido a bateria, motorizado, que gera movimento } \\
\text { ativo nas articulações do quadril e joelho, de maneira } \\
\text { adequadamente sequenciada } \\
\text { Número total de sessões: } 18\end{array}$ & $\begin{array}{l}\text { A DMO foi medida no total e na perna } \\
\text { com DXA e na tíbia com pQTC }\end{array}$ & $\begin{array}{l}\text { Apenas } 1 \text { participante que iniciou o estudo com } \\
\text { diagnóstico de osteoporose melhorou seu status para } \\
\text { osteopenia. Na avaliação da perna com DXA, houve } \\
\text { uma melhora de } 5 \% \text { na DMO. Na avaliação da tibia } \\
\text { com TC, houve uma melhora de } 14,5 \% \text { na DMO }\end{array}$ \\
\hline
\end{tabular}

Absorciometria de raios X de dupla energia (DXA); Activity based therapy (ABT); densidade mineral óssea (DMO); Eletroestimula ção (EE); Eletroestimulação funcional (FES); Exoesqueleto Robótico Ekso (Ekso), Frequência cardíaca (FC); High-resolution peripheral quantitative computed tomography (HRpQCT); tomografia computadoriza (TC); Tomografia computadorizada de alta resolução (TCAR) 


\section{Quadro 4. Limitações dos Estudos}

\begin{tabular}{|c|c|}
\hline Autor & Limitações dos Estudos \\
\hline $\begin{array}{l}\text { Craven et al. }{ }^{21} \\
2017\end{array}$ & $\begin{array}{l}\text { - Perda de } 17 \% \text { dos participantes } \\
\text { - Pacientes tetraplégicos e paraplégicos ambos incompletos (AIS C e D). Não descreve grau de funcionalidade, comorbidades e exposição aos fatores } \\
\text { risco para osteopenia/osteoporose dos participantes } \\
\text { - Alguns indivíduos fizeram uso concomitante de vitamina D, cálcio, bifosfonatos, fatores que podem alterar o resultado da DMO avaliada } \\
\text { - Não é possível afirmar a magnitude do efeito }\end{array}$ \\
\hline $\begin{array}{l}\text { Arija-Blázquez et al. }{ }^{22} \\
2014\end{array}$ & $\begin{array}{l}\text { - Perdas de } 33 \% \text { dos participantes } \\
\text { - № limitado de pacientes ( } 12 \text { pacientes, sendo que apenas } 8 \text { finalizaram o estudo) } \\
\text { - Variabilidade de idade entre os pacientes (18 a } 55 \text { anos) } \\
\text { - Pacientes com lesões recentes (<8 semanas) com maior velocidade de perda, todos do sexo masculino. Dessa forma, há uma dificuldade de extrapolar } \\
\text { os resultados para pacientes do sexo feminino } \\
\text { - Não descreve comorbidades dos participantes e exposição aos fatores de risco para osteopenia/osteoporose }\end{array}$ \\
\hline $\begin{array}{l}\text { Menéndez et al. } .^{23} \\
2016\end{array}$ & $\begin{array}{l}\text { - Apenas } 29 \% \text { dos participantes eram do sexo feminino, sendo esse o grupo com maior fator de risco para perda de DMO } \\
\text { - Pacientes tetraplégicos e paraplégicos ambos completos (AIS A e B) } \\
\text { - Avaliado tempo longo pós lesão (média de } 13,3 \% \text { de lesão). Questionável se pacientes apresentaram tanta perda de DMO que não respondem mais a } \\
\text { intervenção } \\
\text { - Tempo curto de estimulação ( } 10 \mathrm{~min} \text { ) } \\
\text { - Tempo insuficiente para repetir DXA (12 semanas) }\end{array}$ \\
\hline $\begin{array}{l}\text { Dudley-Javoroski et al. }{ }^{24} \\
2012\end{array}$ & $\begin{array}{l}\text { - Não especificado número de perdas ao longo do estudo } \\
\text { - Grande variabilidade na idade dos participantes ( } 16 \text { a } 64 \text { anos) e de tempo pós-lesão }(0,19 \text { a } 24,23 \text { anos) } \\
\text { - Participantes tetraplégicos e paraplégicos completos (AIS A e B), porém não descreve grau de funcionalidade dos participantes } \\
\text { - Apenas } 19 \% \text { dos participantes eram do sexo feminino, sendo esse o grupo com maior fator de risco para perda de DMO } \\
\text { - Alta intensidade de eletroestimulação, porém difícil manter tal intensidade após o estudo } \\
\text { - A adesão dos pacientes na realização da intervenção proposta não apresentou adesão de } 100 \% \text { nos grupos, sendo apenas } 67,6 \% \text { no grupo } 1 \text { e } 66 \% \text { no } \\
\text { grupo } 2 \\
\text { - O autor particiona o tempo de lesão em múltiplos grupos para facilitar a análise, porém as múltiplas divisões com um número pequeno de participantes } \\
\text { acabam dificultando a análise }\end{array}$ \\
\hline $\begin{array}{l}\text { Groah et al. }{ }^{25} \\
2010\end{array}$ & $\begin{array}{l}\text { - Perdas de } 16 \% \text { dos participantes } \\
\text { - Apenas } 15 \% \text { dos participantes eram do sexo feminino, sendo esse o grupo com maior fator de risco para perda de DMO } \\
\text { - Participantes tetraplégicos e paraplégicos completos (AIS A e B), porém não descreve o grau de funcionalidade } \\
\text { - Tempo pós-lesão muito recente }(<12 \text { sem) e tempo de seguimento insuficiente ( } 3 \text { meses) }\end{array}$ \\
\hline $\begin{array}{l}\text { Lai et al. }{ }^{26} \\
2010\end{array}$ & $\begin{array}{l}\text { - Grande variabilidade na idade dos pacientes ( } 20 \text { a } 51 \text { anos) } \\
\text { - Apenas } 14 \% \text { dos participantes eram do sexo feminino, sendo esse o grupo com maior fator de risco para perda de DMO } \\
\text { - Tempo recente de lesão medular para avaliar intervenção ( } 26 \text { a } 52 \text { dias após lesão medular) } \\
\text { - Não relatado atividade realizada pelo grupo controle } \\
\text { - Tempo insuficiente para repetir DXA ( } 3 \text { meses) }\end{array}$ \\
\hline $\begin{array}{l}\text { Morse et al. }{ }^{27} \\
2019\end{array}$ & $\begin{array}{l}\text { - Alta taxa de perdas de participantes ( } 52 \%) \\
\text { - Apenas } 10 \% \text { dos participantes eram do sexo feminino, sendo esse o grupo com maior fator de risco para perda de DMO } \\
\text { - Grande variabilidade no nível de lesão medular dos pacientes. Estudados pacientes completos e incompletos (AIS A, B ou C) e, também, não descreve } \\
\text { grau de funcionalidade dos participantes } \\
\text { - Inicialmente seria realizada nos momentos que a realização de vQCT, porém o centro de coleta de DXA se retirou do estudo } \\
\text { - Não é possível afirmar a magnitude do efeito }\end{array}$ \\
\hline $\begin{array}{l}\text { Dudley Javoroski et al. }{ }^{28} \\
2016\end{array}$ & $\begin{array}{l}\text { - Perda de } 14 \% \text { dos pacientes e } n \text { o pequeno de participantes }(\mathrm{N}=7) \\
\text { - Estudo sem grupo controle } \\
\text { - Grande variabilidade na idade dos pacientes ( } 17 \text { a } 66 \text { anos) e no tempo pós lesão }(0,1 \text { a } 29,2 \text { anos após lesão medular) } \\
\text { - Apenas } 28 \% \text { dos participantes eram do sexo feminino, sendo esse o grupo com maior fator de risco para perda de DMO } \\
\text { - Adesão dos participantes em relação a dose de treinamento recomendada ( } 3 \text { sessões/semana) foi de } 76,9 \%\end{array}$ \\
\hline $\begin{array}{l}\text { Edwards et al. }{ }^{29} \\
2018\end{array}$ & $\begin{array}{l}\text { - Apenas } 22,9 \% \text { dos participantes eram do sexo feminino, sendo esse o grupo com maior fator de risco para perda de DMO } \\
\text { - Participantes tetraplégicos e paraplégicos completos e incompletos (AIS A, B, C e D), porém não descreve o grau de funcionalidade de cada participante } \\
\text { - Grande variabilidade no nível de lesão medular (cervical, torácico e lombar), não sendo homogêneo o número de participantes com lesão lombar entre } \\
\text { os grupos (apenas o grupo } 1 \text { apresenta } 2 \text { participantes com lesão lombar, os demais grupos não possuem participantes com esse nível de lesão) } \\
\text { - Baixa intensidade de intervenção - sessões de apenas } 10 \text { minutos de vibração/ dia } \\
\text { - Os resultados baseados no exame TC não passaram no controle de qualidade, devido a falta de dados na linha de base ou após } 12 \text { meses de intervenção, } \\
\text { artefatos, movimentos ou metal e desvio do protocolo de TC } \\
\text { - Chama a atenção o volume de perda para pacientes com esta cronicidade }\end{array}$ \\
\hline $\begin{array}{l}\text { Wuermser et al. } .^{30} \\
2015\end{array}$ & $\begin{array}{l}\text { - Perda de } 33 \% \text { ( } 3 \text { perdas antes da } 1 \text { avaliação e } 1 \text { após) } \\
\text { - Estudo sem grupo controle } \\
\text { - Baixa intensidade de intervenção - sessões de apenas } 20 \text { minutos de ortostatismo } \\
\text { - Grande variabilidade no tempo pós lesão ( } 2 \text { a } 27 \text { anos após lesão medular - não discriminado entre todos os participantes) } \\
\text { - Apesar do estudo avaliar o efeito da vibração, é relatado que alguns indivíduos permaneceram na plataforma vibratória por um período maior, de } 20 \text { - } \\
60 \text { minutos, que outros pacientes. No entanto, é importante salientar que o ortostatismo realizado pelos participantes durante o tempo excedido } \\
\text { comparado aos outros pacientes, interfere no resultado do estudo }\end{array}$ \\
\hline $\begin{array}{l}\text { Astorino et al. }{ }^{31} \\
2013\end{array}$ & $\begin{array}{l}\text { - Perdas de } 23 \% \text { dos participantes } \\
\text { - Apenas } 15 \% \text { dos participantes eram do sexo feminino, sendo esse o grupo com maior fator de risco para perda de DMO } \\
\text { - Não tem grupo controle } \\
\text { - Grande Variabilidade de nível de lesão medular: Participantes com lesão abaixo do nível C2 completos e incompletos (sem determinação da } \\
\text { classificação AIS), bem como não descreve grau de funcionalidade dos participantes } \\
\text { - Grande variabilidade no tempo pós lesão (<3 meses e > } 3 \text { meses após lesão medular) e na intensidade das intervenções: variação de } 4,6 \text { a } 13 \text { horas } \\
\text { por semana entre os participantes } \\
\text { - Múltiplas intervenções analisadas concomitantemente }\end{array}$ \\
\hline $\begin{array}{l}\text { Chain et al. }{ }^{32} \\
2012\end{array}$ & $\begin{array}{l}\text { - Participantes tetraplégicos (nível C5 a C7), com lesão medular completa e incompleta (sem determinação da classificação AIS), sem determinação do } \\
\text { nível de funcionalidade dos participantes } \\
\text { - Grande variabilidade no tempo pós lesão ( } 1 \text { a } 24 \text { anos após lesão medular) } \\
\text { - Não discrimina o sexo dos participantes do estudo e não determina momento de medição de DXA }\end{array}$ \\
\hline $\begin{array}{l}\text { Karelis et al. }{ }^{33} \\
2017\end{array}$ & $\begin{array}{l}\text { - Apenas } 20 \% \text { dos participantes eram do sexo feminino, sendo esse o grupo com maior fator de risco para perda de DMO } \\
\text { - Não tem grupo controle } \\
\text { - Participantes idosos, com uma média de idade de } 60,4 \text { anos } \\
\text { - № insuficiente de participantes ( } N=5) \text { para que possamos extrapolar os seus resultados para todos os pacientes com lesão medular } \\
\text { - Estudo realizado por um período de tempo curto ( } 6 \text { semanas) } \\
\text { - Tempo insuficiente para avaliar alteração de DMO ( } 6 \text { semanas) } \\
\text { - Participantes com lesão medular crônica (média de } 7,6 \text { anos após lesão) }\end{array}$ \\
\hline
\end{tabular}




\section{DISCUSSÃO}

\section{Eletroestimulação}

Foram encontrados 7 artigos que estudaram o FES associado ou não a outra intervenção. Craven et al. ${ }^{21}$ e ArijaBlázquez et al. ${ }^{22}$ avaliaram unicamente o FES como intervenção, sendo que o primeiro avaliou caminhada assistida + FES durante 45 minutos, 3x/semana durante 4 meses e o último avaliou FES durante 47 minutos, 5x/semana durante 14 semanas. Ambos concluíram não haver diferença significativa na DMO pré e pós-intervenção. Craven et al. ${ }^{21}$ levantou a hipótese da ausência de melhora da DMO ser decorrente do tempo da intervenção de apenas 4 meses, visto que a renovação óssea tem duração de pelo menos 3 a 6 meses.

Menéndez et al. ${ }^{23}$ analisaram o efeito da EE associada a vibração na DMO, corroborando com os achados descritos acima de ausência de melhora na DMO. Porém o estudo apresenta como limitações o fato de ter avaliado múltiplos desfechos e não ter especificado a atividade realizada pelo grupo controle.

Dudley-Javoroski et al. ${ }^{24}$ estudaram o efeito da eletroestimulação em quadríceps conjunta com ortostatismo e concluíram o declínio nas taxas de perdas da DMO do fêmur distal. Foi observado diminuição da inclinação da curva de perda de DMO nesta região ao longo do tempo de cerca de 3 vezes maior para o grupo 1 em relação ao grupo 2 e 3 . Apesar de não ter evidenciado ganhos, o fato de ter diminuído a taxa de perda óssea é considerado um efeito benéfico da intervenção.

Assim como Arija-Blázquez et al. ${ }^{22}$, Lai et al. ${ }^{26}$ e Groah et al. ${ }^{25}$ avaliaram o efeito do FES combinada ou não a outras intervenções em pacientes com lesão aguda, diferentemente dos demais que avaliaram grupos de pacientes lesões crônicas ou grupos mistos.

Groah et al. ${ }^{25}$ avaliaram o efeito da atividade física associada a EE e concluíram que a EE pode limitar a perda óssea no período agudo após a LM. Como a EE foi aplicada ao quadríceps, a maior melhora seria esperada sobre o fêmur distal. Portanto, embora os dois grupos tenham apresentado perda de DMO na lombar e quadril, o grupo intervenção teve $50 \%$ menos (embora não seja estatisticamente significativo) perda distal da DMO do fêmur. Além disso, parecia haver uma tendência à redução da perda de DMO sobre a tíbia proximal, embora o efeito não tenha sido tão forte quanto no fêmur distal. Isso é esperado, pois a área da superfície do quadríceps é maior sobre o fêmur que a tíbia, resultando em maior força aplicada ao fêmur durante a contração do músculo quadríceps.

Lai et al. ${ }^{26}$ avaliaram o efeito do FES-bike concluindo ter havido redução parcial da massa óssea em fêmur distal. A DMO no colo do fêmur e fêmur distal diminuiu significativamente entre a primeira e a segunda medidas nos grupos FESCE e controle, com atenuação da redução da DMO no fêmur distal, mas não no colo do fêmur durante o período de FESCE. Os autores sugerem que mais estudos são necessários para determinar se redução adicional da perda óssea poderia ser obtida por resistência ao ciclismo ou treinamento mais frequente que o usado neste estudo.

Morse et al. ${ }^{27}$ analisaram o efeito do FES + remo + ácido zolendrônico (AZ) e concluíram que no grupo intervenção houve incremento de DMO em maior proporção que o grupo controle. $O$ estudo acima foi incluído nesta revisão sistemática objetivando os resultados do grupo controle, porém como não foi estudado um grupo sem qualquer intervenção, não foi possível concluir se houve diferença na DMO apenas com FES + remo. Além disso, o trabalho em questão apresentou grande perda de participantes durante o seguimento (44\% de perdas no grupo controle e $50 \%$ de perdas no grupo intervenção), mesmo que não relacionadas a efeitos adversos da intervenção.

$\mathrm{Na}$ revisão sistemática realizada por Biering-Sørensen et al. ${ }^{20}$ que foi usada como base para este estudo, foi avaliado o efeito do FES na DMO e concluiu que a melhora na DMO foi evidenciada naqueles estudos com maior tempo de treinamento, maior frequência e maior intensidade do estímulo. Após a experiência adquirida com os estudos, a recomendação é que a estimulação elétrica deve ser realizada pelo menos 2 a 3 vezes por semana e, provavelmente, deva ser continuada a longo prazo para que a massa óssea não diminua ainda mais.

Na revisão sistemática e metanálise publicada por Chang et al. ${ }^{34}$, foram estudados o efeito de bifosfonados e/ou FES na DMO. Apesar dos estudos demonstrarem benefícios em relação a DMO, o tempo de seguimento foi insuficiente para avaliar os efeitos das intervenções na prevenção de fraturas relacionadas a osteoporose. Além disso, o presente estudo levantou a questão de a heterogeneidade dos participantes nos quesitos sexo, tempo e nível de lesão, influenciarem na significância dos resultados encontrados. Apesar disso, os autores concluem que o FES pode aumentar significativamente a DMO sublesional adjacente ao local de carga mecânica máxima em pacientes com LM crônica e sendo que treinos com no mínimo 5 dias por semana foram associados a maior eficácia. ${ }^{35}$

$\mathrm{Na}$ revisão sistemática de Soleyman-Jahi et al. ${ }^{13}$, foram encontrados 27 artigos sobre o efeito das intervenções não farmacológicas na DMO de indivíduos com $L M$, sendo que apenas 1 era ECR e 1 estudo clínico não randomizado. Os autores concluíram que ciclismo + FES (3 ou menos vezes por semana), não melhoram ou evitam perda óssea em indivíduos com mais de um ano de LM. No entanto, existem evidências de estudos não randomizados que mostram que o ciclismo + FES (5 ou mais vezes por semana), pode ser eficaz na prevenção da perda de massa óssea.

\section{Plataforma vibratória}

Dudley-Javoroski et al. ${ }^{28}$, Edwards et al. ${ }^{29}$ e Wuermser et al. $^{30}$ avaliaram o efeito da vibração na DMO e não houve evidência de incremento na massa óssea após a intervenção. Entretanto, Wuermser et al. ${ }^{30}$ evidenciaram diminuição na taxa de perda óssea no quadril, sendo este considerado um resultado positivo. Importante pontuar que neste estudo o grupo era homogêneo em relação ao sexo, diferentemente dos outros estudos em que há discrepância tendenciosa para o sexo masculino. Vale ressaltar, que Edwards et al. ${ }^{29}$ avaliaram o efeito da vibração combinada ou não a teriparatida, sendo o resultado do grupo controle o material de estudo analisado nesta revisão sistemática. 
Astorino et al. ${ }^{31}$ também avaliaram o efeito da vibração, porém combinado a atividade física, FES, bicicleta ergométrica e marcha (ativa e passiva). Este estudo avaliou múltiplas intervenções não sendo possível avaliar a influência isolada de cada uma delas. Além disso, não há grupo controle e não foi especificado quais foram as atividades físicas realizadas.

$\mathrm{Na}$ revisão sistemática realizada por Biering-Sørensen et al. ${ }^{20}$ foi incluído somente 1 estudo que avaliou vibração, que evidenciou melhora na DMO, porém era um estudo de baixa qualidade e com apenas 2 indivíduos.

\section{Atividade física}

Chain et al. ${ }^{32}$ realizaram um estudo onde foi avaliado o efeito da atividade física habitual na DMO de tetraplégicos. Os resultados encontrados sugerem que o início precoce do exercício físico após a ocorrência de lesão pode ajudar a atenuar a desmineralização do osso fêmur em homens com LM cervical. Porém, não foram estipulados os tipos de exercícios físicos realizados, apenas frequência dos treinos, havendo alto risco de viés de intervenção. Além disso, foram consideradas apenas 1 medida de DXA, não sendo possível avaliar se houve ou não melhora na DMO.

\section{Robótica}

Karelis et al. ${ }^{33}$ fizeram o primeiro estudo a indicar treinamento locomotor usando exoesqueleto robótico. Porém a intervenção estudada foi por apenas 6 semanas, com poucos pacientes e sem grupo controle. Os autores discutiram sobre evidência de melhora da DMO em 14,5\% na tíbia, porém vale ressaltar que o $p$-value do parâmetro analisado era de 0,08 (superior a 0,05), sendo assim não há significância estatística no resultado encontrado. Além disso, esse resultado foi baseado nos exames de apenas 1 indivíduo e o restante dos resultados foram apresentadas em conjunto. Desta forma, ainda não é possível extrapolar esses resultados para a prática médica.

\section{Limitações dos Estudos}

Observa-se que há um número pequeno de estudos que englobam intervenções não farmacológicas na densidade mineral óssea e, ainda assim, os estudos apresentam múltiplas limitações como ausência de grupo controle, lesões medulares de diferentes níveis e tempos de lesão, além de pequeno número de participantes sendo estes em sua maioria do sexo masculino. Sendo assim, na tabela 4 encontram-se descritas as limitações encontradas nos estudos analisados, corroborando a dificuldade de comparar os estudos consistentemente.

\section{CONCLUSÃO}

As evidências dos uso de terapias não farmacológicas para prevenção e tratamento da osteoporose em LM são fracas e seus estudos contem inúmeros vieses (de seleção, performance, detecção, atrito, relato, além de ausência de grupo controle, número insuficiente de participantes e grande número de perdas ao longo do estudo) impossibilitando tirar conclusões mais robustas. Consideramos que primeiramente os estudos devem separar grupos para a prevenção de perda de massa óssea (até 1 ano após lesão ou idealmente antes de 6 meses) e grupos para estabilização ou ganho de massa óssea (pacientes com lesão com mais de 1 ano).

Os pacientes para estudo devem ser alocados em grupos homogêneos que levem em consideração o sexo, o nível de lesão, a classificação AIS (estudando separadamente pacientes A e $B$ dos incompletos motores), nível de independência funcional $^{35}$ (que não foi considerado em nenhum estudo analisado), classificação de grau de deambulação, ${ }^{36}$ outros fatores de risco para osteoporose e uso de medicações que modificam o metabolismo ósseo. Vale ainda destacar que estudos que avaliem a diminuição de risco de fratura não foram encontrados.

Levando em consideração todas as intervenções não farmacológicas apresentadas, destacamos que o FES é a modalidade mais estudada, com mais trabalhos disponibilizados nas bases de dados, porém, ainda assim, com baixo nível de evidência. Consideramos que o uso de FES pode ser benéfico tanto para prevenção como para tratamento da perda de massa óssea. Os resultados são observados com um uso frequente ( 5 sessões semanais) e associado a atividades físicas em especial as que promovam maior resistência muscular. Destacamos também que o retardo na perda de massa óssea está circunscrito ao período de aplicação, cessando após o término do mesmo. No entanto, vale dizer que maiores esclarecimentos sobre os músculos a serem estimulados, os protocolos de eletroestimulação, o momento ideal de início do tratamento bem como a duração do mesmo precisam ser investigados.

A associação de eletroestimulação e exercícios parece potencializar a ação medicamentosa, mas mais estudos são necessários para ratificar esta impressão. O treino de marcha robótico também parece ter potencial de ação benéfico na massa óssea, mas as evidências ainda são incipientes. Não foram encontradas evidências que justifiquem no momento o uso de plataforma vibratória.

Acreditamos que a osteoporose em LM é multifatorial e um melhor resultado de tratamento deva ser obtido com modalidades de tratamento multimodais (tratamento farmacológico e não farmacológico) e a pesquisa dos mesmos deva ser fortemente incentivada.

Intervenções não farmacológicas como o ortostatismo, atividades físicas, treino de marcha, eletroestimulação são estratégias de baixo custo, baixo risco, poucos efeitos colaterais e com inúmeros outros benefícios no tratamento e reabilitação de lesados medulares. Por isso, ainda que não tenhamos evidências consistentes de ação na massa óssea, estão fortemente recomendados dentro do rol de atividades desta população.

Em relação a revisão sistemática de Biering-Sørensen et al. $^{20}$ pouco evoluímos em relação ao tratamento não farmacológico de osteoporose em LM. Isto reforça a necessidade de investimento em mais estudos e com melhor qualidade nesta área.

\section{REFERENCIAS}

1. Spinal cord injury facts and figures at a gla nce. J Spinal Cord Med. 2012;35(4):197-8. Doi: https://doi.org/10.1179/1079026812Z.00000000063 
2. Brasil. Ministério da Saúde. Diretrizes de atenção à pessoa com lesão medular. 2 ed. Brasília (DF): Ministério da Saúde; 2015.

3. Dionyssiotis $\mathrm{Y}$, Stathopoulos $\mathrm{K}$, Trovas G, Papaioannou N, Skarantavos G, Papagelopoulos P. Impact on bone and muscle area after spinal cord injury. Bonekey Rep. 2015;4:633.

https://doi.org/10.1038/bonekey.2014.128

Doi:

4. Mazwi NL, Adeletti K, Hirschberg RE. Traumatic Spinal Cord Injury: Recovery, Rehabilitation, and Prognosis. Curr Trauma Rep. 2015;1(3):182-92. Doi: https://doi.org/10.1007/s40719-015-0023-x

5. Hammond ER, Metcalf HM, McDonald JW, Sadowsky CL. Bone mass in individuals with chronic spinal cord injury: associations with activity-based therapy, neurologic and functional status, a retrospective study. Arch Phys Med Rehabil. 2014;95(12):2342-9. Doi: https://doi.org/10.1016/j.apmr.2014.07.395

6. Hochberg MC, Silman AJ, Smolen JS, Weinblatt ME, Weisman MH. Reumatologia. 6 ed. Rio de Janeiro: Elsevier; 2016.

7. Jiang SD, Jiang LS, Dai LY. Mechanisms of osteoporosis in spinal cord injury. Clin Endocrinol (Oxf). 2006;65(5):555-65. Doi: https://doi.org/10.1111/j.1365-2265.2006.02683.x

8. Maïmoun L, Fattal C, Micallef JP, Peruchon E, Rabischong P. Bone loss in spinal cord-injured patients: from physiopathology to therapy. Spinal Cord. 2006;44(4):20310. Doi: https://doi.org/10.1038/sj.sc.3101832

9. Haider IT, Lobos SM, Simonian N, Schnitzer TJ, Edwards WB. Bone fragility after spinal cord injury: reductions in stiffness and bone mineral at the distal femur and proximal tibia as a function of time. Osteoporos Int. 2018;29(12):2703-15. Doi: https://doi.org/10.1007/s00198-018-4733-0

10. Dionyssiotis Y, Lyritis GP, Mavrogenis AF, Papagelopoulos PJ. Factors influencing bone loss in paraplegia. Hippokratia. 2011;15(1):54-9.

11. Maïmoun L, Fattal C, Sultan C. Bone remodeling and calcium homeostasis in patients with spinal cord injury: a review. Metabolism. 2011;60(12):1655-63. Doi: https://doi.org/10.1016/j.metabol.2011.04.005

12. Charmetant $C$, Phaner V, Condemine A, Calmels $P$. Diagnosis and treatment of osteoporosis in spinal cord injury patients: A literature review. Ann Phys Rehabil Med. 2010;53(10):655-68. Doi: https://doi.org/10.1016/i.rehab.2010.10.001

13. Soleyman-Jahi $S$, Yousefian A, Maheronnaghsh $R$, Shokraneh F, Zadegan SA, Soltani A, et al. Evidence-based prevention and treatment of osteoporosis after spinal cord injury: a systematic review. Eur Spine J. 2018;27(8):1798-1814.

Doi:
14. Fattal C, Mariano-Goulart D, Thomas E, Rouays-Mabit H, Verollet C, Maimoun L. Osteoporosis in persons with spinal cord injury: the need for a targeted therapeutic education. Arch Phys Med Rehabil. 2011;92(1):59-67. Doi: https://doi.org/10.1016/i.apmr.2010.09.019

15. Bauman WA, Cardozo CP. Osteoporosis in individuals with spinal cord injury. PM R. 2015;7(2):188-201. Doi: https://doi.org/10.1016/j.pmrj.2014.08.948

16. Cirnigliaro CM, Myslinski MJ, La Fountaine MF, Kirshblum SC, Forrest GF, Bauman WA. Bone loss at the distal femur and proximal tibia in persons with spinal cord injury: imaging approaches, risk of fracture, and potential treatment options. Osteoporos Int. 2017;28(3):747-65. Doi: https://doi.org/10.1007/s00198-016-3798-x

17. Troy KL, Morse LR. Measurement of Bone: Diagnosis of $\mathrm{SCl}-$ Induced Osteoporosis and Fracture Risk Prediction. Top Spinal Cord Inj Rehabil. 2015;21(4):267-74. Doi: https://doi.org/10.1310/sci2104-267

18. Craven C, Lynch CL, Eng JJ. Bone Health Following Spinal Cord Injury. In: Eng JJ, Teasell RW, Miller WC, Wolfe DL, Townson AF, Hsieh JTC, et al. Spinal Cord Injury Rehabilitation Evidence. Version 5.0. Vancouver: SCIRE; 2019. p 1-37.

19. Battaglino RA, Lazzari AA, Garshick E, Morse LR. Spinal cord injury-induced osteoporosis: pathogenesis and emerging therapies. Curr Osteoporos Rep. 2012;10(4):278-85. Doi: https://doi.org/10.1007/s11914012-0117-0

20. Biering-Sørensen F, Hansen B, Lee BS. Nonpharmacological treatment and prevention of bone loss after spinal cord injury: a systematic review. Spinal Cord. 2009;47(7):508-18.

Doi: https://doi.org/10.1038/sc.2008.177

21. Craven BC, Giangregorio LM, Alavinia SM, Blencowe LA, Desai N, Hitzig SL, et al. Evaluating the efficacy of functional electrical stimulation therapy assisted walking after chronic motor incomplete spinal cord injury: effects on bone biomarkers and bone strength. J Spinal Cord Med. 2017;40(6):748-58. Doi: https://doi.org/10.1080/10790268.2017.1368961

22. Arija-Blázquez $A$, Ceruelo-Abajo $S$, Díaz-Merino $M S$, Godino-Durán JA, Martínez-Dhier L, Martin JL, et al. Effects of electromyostimulation on muscle and bone in men with acute traumatic spinal cord injury: A randomized clinical trial. J Spinal Cord Med. 2014;37(3):299-309.

https://doi.org/10.1179/2045772313Y.0000000142

23. Menéndez $H$, Ferrero $C$, Martín-Hernández J, Figueroa $A$, Marín PJ, Herrero AJ. Chronic effects of simultaneous electromyostimulation and vibration on leg blood flow in spinal cord injury. Spinal Cord. 2016;54(12):1169-75. Doi: https://doi.org/10.1038/sc.2016.60 
24. Dudley-Javoroski S, Saha PK, Liang G, Li C, Gao Z, Shields RK. High dose compressive loads attenuate bone mineral loss in humans with spinal cord injury. Osteoporos Int. 2012;23(9):2335-46.

Doi: https://doi.org/10.1007/s00198-011-1879-4

25. Groah SL, Lichy AM, Libin AV, Ljungberg I. Intensive electrical stimulation attenuates femoral bone loss in acute spinal cord injury. PM R. 2010;2(12):1080-7. Doi: https://doi.org/10.1016/i.pmri.2010.08.003

26. Lai $\mathrm{CH}$, Chang WH, Chan WP, Peng CW, Shen LK, Chen JJ, et al. Effects of functional electrical stimulation cycling exercise on bone mineral density loss in the early stages of spinal cord injury. J Rehabil Med. 2010;42(2):150-4. Doi: https://doi.org/10.2340/16501977-0499

27. Morse LR, Troy KL, Fang Y, Nguyen N, Battaglino R, Goldstein RF, et al. Combination therapy with zoledronic acid and fes-row training mitigates bone loss in paralyzed legs: results of a randomized comparative clinical trial. JBMR Plus. 2019;3(5):e10167. Doi: https://doi.org/10.1002/jbm4.10167

28. Dudley-Javoroski S, Petrie MA, McHenry CL, Amelon RE, Saha PK, Shields RK. Bone architecture adaptations after spinal cord injury: impact of long-term vibration of a constrained lower limb. Osteoporos Int. 2016;27(3):114960. Doi: https://doi.org/10.1007/s00198-015-3326-4

29. Edwards WB, Simonian N, Haider IT, Anschel AS, Chen D, Gordon KE, et al. Effects of teriparatide and vibration on bone mass and bone strength in people with bone loss and spinal cord injury: a randomized, controlled trial. J Bone Miner Res. 2018;33(10):1729-1740. Doi: https://doi.org/10.1002/jbmr.3525

30. Wuermser LA, Beck LA, Lamb JL, Atkinson EJ, Amin S. The effect of low-magnitude whole body vibration on bone density and microstructure in men and women with chronic motor complete paraplegia. J Spinal Cord Med. 2015;38(2):178-86.

Doi:
31. Astorino TA, Harness ET, Witzke KA. Effect of chronic activity-based therapy on bone mineral density and bone turnover in persons with spinal cord injury. Eur J Appl Physiol. 2013;113(12):3027-37. doi: https://doi.org/10.1007/s00421-013-2738-0

32. Chain A, Koury JC, Bezerra FF. Physical activity benefits bone density and bone-related hormones in adult men with cervical spinal cord injury. Eur J Appl Physiol. 2012;112(9):3179-86.

Doi: https://doi.org/10.1007/s00421-011-2303-7

33. Karelis AD, Carvalho LP, Castillo MJ, Gagnon DH, AubertinLeheudre $M$. Effect on body composition and bone mineral density of walking with a robotic exoskeleton in adults with chronic spinal cord injury. J Rehabil Med. 2017;49(1):84-87.

Doi: https://doi.org/10.2340/16501977-2173

34. Chang KV, Hung CY, Chen WS, Lai MS, Chien KL, Han DS. Effectiveness of bisphosphonate analogues and functional electrical stimulation on attenuating post-injury osteoporosis in spinal cord injury patients- a systematic review and meta-analysis. PLoS One. 2013;8(11):e81124. Doi: https://doi.org/10.1371/journal.pone.0081124

35. Ackerman P, Morrison SA, McDowell S, Vazquez L. Using the Spinal Cord Independence Measure III to measure functional recovery in a post-acute spinal cord injury program. Spinal Cord. 2010;48(5):380-7. Doi: https://doi.org/10.1038/sc.2009.140

36. Dittuno PL, Ditunno JF Jr. Walking index for spinal cord injury (WISCl II): scale revision. Spinal Cord. 2001;39(12):654-6. Doi: 